\title{
Ubiquitin ligase $R N F 5$ serves an important role in the development of human glioma
}

\author{
YONG GAO $^{1 *}$, CHENGMIN XUAN $^{2 *}$, MINGWEI JIN $^{2 *}$, QI AN $^{2}$, BAOBIAO ZHUO $^{1}$, \\ XINCHENG CHEN $^{3}$, LEI WANG ${ }^{2}$, YUAN WANG ${ }^{2}$, QINGZENG SUN ${ }^{1}$ and YINGCHUN SHI ${ }^{1}$
}

\author{
Departments of ${ }^{1}$ Orthopedics and ${ }^{2}$ Hematology, Xuzhou Children's Hospital of Xuzhou Medical University, Xuzhou, \\ Jiangsu 221006; ${ }^{3}$ Department of Neurosurgery, Xinyi People's Hospital, Xinyi, Jiangsu 221400, P.R. China
}

Received January 7, 2019; Accepted August 6, 2019

DOI: $10.3892 / \mathrm{ol} .2019 .10801$

\begin{abstract}
The ubiquitin ligase ring finger protein 5 (RNF5) has previously been associated with the development of breast cancer. Patients with breast cancer and high RNF5 expression have been demonstrated to have a shorter survival time compared with patients with low RNF5 expression. However, the role of RNF5 in human glioma has not been determined. The present study analyzed the role of RNF5 in gliomas using bioinformatics analysis. The results revealed that RNF5 was differentially expressed in non-cancerous brain tissues and different grades of glioma. Furthermore, a high RNF5 expression in patients with glioma was associated with an improved prognosis compared with patients with low expression. Gene Set Enrichment Analysis revealed that RNF5 was particularly associated with 'Wnt signaling pathway', 'apoptosis', 'focal adhesion' and 'cytokine-cytokine receptor interaction' in patients with glioma. Additionally, 4 potential ubiquitination substrates for RNF5 were predicted, including sorting nexin 10 , proprotein convertase subtilisin/kexin type 1 , leucine rich glioma inactivated 1 and solute carrier family 39 member 12. These findings provided the basis for further investigation on the role of $R N F 5$ in tumors.
\end{abstract}

\section{Introduction}

Gliomas are the most common primary brain tumors with an incidence rate of $~ 5 / 100,000)$. Despite comprehensive treatment strategies, including surgery, radiotherapy and chemotherapy, the prognosis of patients remains unsatisfactory, with a median

Correspondence to: Dr Yingchun Shi or Professor Qingzeng Sun, Department of Orthopedics, Xuzhou Children's Hospital of Xuzhou Medical University, 18 Sudi Road, Xuzhou, Jiangsu 221006, P.R. China E-mail: shiyc6025@163.com

E-mail: sqzjyym@163.com

*Contributed equally

Key words: ring finger protein 5, glioma, bioinformatics, differentially expressed genes, ubiquitination survival time of $12-18$ months (1-4). This poor outcome is largely associated with the difficulty of curing gliomas and the high relapse rates $(5,6)$. Therefore, the identification of novel therapeutic targets has become a particular focus of research.

Ring finger protein 5 (RNF5) belongs to the ring finger family of ubiquitin ligases $(7,8)$, which are anchored to the endoplasmic reticulum (ER) membrane and are important components of the ER-associated degradation (ERAD) mechanism. RNF5 serves a role in monitoring the folding of CF transmembrane conductance regulator (CFTR), CFTRDF508 and nascent CFTR $\Delta$ F508 in the ER membrane $(9,10)$. Furthermore, a previous study reported that $R N F 5$ regulates cell movement by targeting paxillin ubiquitination and altering its localization (11). RNF5 participates in the inflammatory response in viral infections by ubiquitinating transmembrane protein 173 and inhibiting the activation of virus-induced interferon regulatory factor 3 , expression of interferon $\beta 1$ and the cellular antiviral response (12). In breast cancer cells, RNF5 ubiquitination degrades the L-glutamine carrier proteins solute carrier family 1 member 5 and solute carrier family 38 member 2 , which reduces glutamine uptake and levels of the tricarboxylic acid cycle components, decreases mechanistic target of rapamycin signaling and cell proliferation, and increases autophagy and apoptosis (13). RNF5 is highly expressed in breast cancer and related cell lines, and inhibition of its expression decreases cell proliferation (13). The present study attempted to characterize the role of RNF5 in human glioma and to determine its association with tumor grade and survival time in patients with glioma.

The present study revealed that RNF5 was differentially expressed in patients with different grades of glioma and was closely associated with the prognosis of patients with anaplastic glioma (AG) and glioblastoma multiforma (GBM). Moreover, Gene Set Enrichment Analysis (GSEA) identified the Kyoto Encyclopedia of Genes and Genomes (KEGG) signaling pathways that were significantly associated with RNF5. Additionally, a correlation analysis was used to predict the potential ubiquitination substrates for RNF5 in human glioma.

\section{Materials and methods}

Patient samples. mRNA microarray expression for patients were obtained from the Chinese Glioma Genome Atlas 
(CGGA; cgga.org.cn) and the Gene Expression Omnibus (www.ncbi.nlm.nih.gov/geo). The CGGA contains 301 glioma samples (including 84 astrocytoma, 89 oligodendroglioma and 128 glioblastoma samples). The grouping of low-grade glioma (LGG), AG and GBM was performed as previously described (14). The GSE16011 dataset (www.ncbi.nlm.nih. gov/geo/query/acc.cgi?acc=GSE16011) contains 276 glioma samples (including samples from 8 patients with epilepsy and 24 astrocytoma, 85 oligodendroglioma and 159 glioblastoma samples) and 8 control samples, totaling 284 specimens (15). The GSE4290 dataset (www.ncbi.nlm.nih.gov/geo/query/acc. cgi?acc=GSE4290) contains 23 samples from patients with epilepsy as non-cancerous samples and 157 tumor samples, including 26 astrocytoma, 50 oligodendrogliomas and 81 glioblastoma samples (16). Gene mutation data were obtained from The Cancer Genome Atlas (TCGA; cancergenome.nih.gov).

RNF5 expression and its association with patient prognosis. The expression levels of RNF5 in the CGGA database and GSE16011 and GSE4290 datasets were analyzed using GraphPad Prism software version 6.01 (GraphPad Software, Inc.). In addition, the association between the expression level of RNF5 and the prognosis of patients was obtained using the CGGA database. In order to analyze patient prognosis, patients were equally divided into two groups according to RNF5 expression levels.

Cell culture. The glioblastoma cell line U251 was obtained from The Type Culture Collection of The Chinese Academy of Sciences. U251 cells were cultured using DMEM (Invitrogen; Thermo Fisher Scientific, Inc.) supplemented with $10 \%$ fetal bovine serum (Gibco; Thermo Fisher Scientific, Inc.) at $37^{\circ} \mathrm{C}$ and $5 \% \mathrm{CO}_{2}$.

Plasmid and transfection. The RNF5 cDNA sequence was inserted between the HindIII and $X b a I$ restriction sites in the p3XFLAG-CMV-14 vector (Shanghai GenePharma Co., Ltd.). The p3XFLAG-CMV-14 plasmid was used as an empty vector. A total of $3 \mu \mathrm{g}$ plasmid was transfected into U251 cells using $9 \mu$ Polyjet transfection reagent (SignaGen Laboratories) according to the manufacturer's protocol. The culture medium was changed after $12 \mathrm{~h}$ and cells were transfected for $72 \mathrm{~h}$.

Reverse transcription-quantitative PCR (RT-qPCR). RNF5 expression was assessed using RT-qPCR. Total RNA was extracted from U251 cells using TRIzol ${ }^{\circledR}$ reagent (Invitrogen; Thermo Fisher Scientific, Inc.) and reverse-transcribed into cDNA using the Quant One-Step RT-PCR kit (Tiangen Biotech Co., Ltd.). qPCR was performed using FastStart Universal SYBR Green Mix (Roche Diagnostics) and an ABI 7300 real-time PCR instrument (Applied Biosystems; Thermo Fisher Scientific, Inc.). The primers for RNF5 and $\beta$-actin were designed as follows: RNF5, forward 5'-GTACCC ATACGATGTTCCAGATTACGC-3', reverse 5'-CTGAGC AGCCAGAAAAAGAAAAAGATG-3'; and $\beta$-actin forward, 5'-CATGTACGTTGCTATCCAGGC-3', and reverse, 5'-CGC TCGGTGAGGATCTTCATG-3'. Thermocycling conditions included pre-denaturation at $95^{\circ} \mathrm{C}$ for $3 \mathrm{~min}$, denaturation at $95^{\circ} \mathrm{C}$ for $15 \mathrm{sec}$, annealing at $60^{\circ} \mathrm{C}$ for $15 \mathrm{sec}$ and extension at $72^{\circ} \mathrm{C}$ for $1 \mathrm{~min}$ for 35 cycles. Expression level of RFN5 was calculated using the $2^{-\Delta \Delta \mathrm{Cq}}$ method (17).

Cell colony formation assay. U251 cells $\left(1 \times 10^{5}\right)$ overexpressing RNF5 were seeded into $6 \mathrm{~cm}$ dishes and cultured for 14 days at $37^{\circ} \mathrm{C}$. Cells were subsequently fixed in $4 \%$ paraformaldehyde for $30 \mathrm{~min}$ at room temperature and stained with $0.05 \%$ crystal violet for $30 \mathrm{~min}$ at room temperature. A light Canon 70D camera (Canon, Inc.) was used to capture images (magnification, $\mathrm{x} 1$ ).

GSEA to evaluate RNF5-enriched KEGG pathways. In order to elucidate the signaling pathways associated with the possible actions of RNF5 in human glioma, enrichment analysis was performed using GSEA software version 6.2 (software.broadinstitute.org/gsea/login.jsp) in the CGGA database. $R N F 5$-enriched KEGG (www.genome.jp/kegg) signaling pathways were identified through this analysis.

Identification of differentially expressed genes (DEGS). To predict the potential substrate(s) of RNF5, CGGA, GSE16011 and GSE4290 data were sorted according to RNF5 expression level from low to high. Data were subsequently divided into four groups: A, B, C and D according to the number of patients following RNF5 determination. To avoid data with no significant differences from groups $\mathrm{B}$ and $\mathrm{C}$, comparative analysis was performed between groups $\mathrm{A}$ and $\mathrm{D}$ to identify the DEGs from the three databases. To narrow the scope, the DEGs that overlapped among the three databases were identified using limma package of R software 3.4.4 (www.r-project.org) and selected for subsequent analysis. A gene with $\mid \log \mathrm{FCl}>1$ was defined as DEG.

Correlation analysis between RNF5 and overlapping genes. To demonstrate the correlation between RNF5 and the five overlapping genes, analysis using $\mathrm{R}$ and GraphPad Prism software was performed. Correlation analysis was performed using Pearson's correlation coefficient test.

Statistical analysis. GraphPad Prism software was used for statistical analysis. Results are presented as the means \pm standard error of the mean. Statistical significance was analyzed using Student's t-test (two groups) and one-way analysis of variance (multiple groups) followed by Dunnett's post hoc test. Kaplan-Meier survival analyses for overall survival were performed and compared with the log-rank test. $\mathrm{P}<0.05$ was considered to indicate a statistically significant difference. For GSEA, a normalized enrichment score $>1$, nominal $\mathrm{P}<0.05$ and false discovery rate q-value $<0.25$ were considered to indicate a statistically significant difference.

\section{Results}

Expression of RNF5 and its association with prognosis. To characterize the expression of RNF5 and its association with patient prognosis, the CGGA database and the GSE16011 and GSE4290 datasets were used. RNF5 was differentially expressed in LGG, AG and GBM. RNF5 expression was significantly higher in LGG and AG compared with GBM 
A
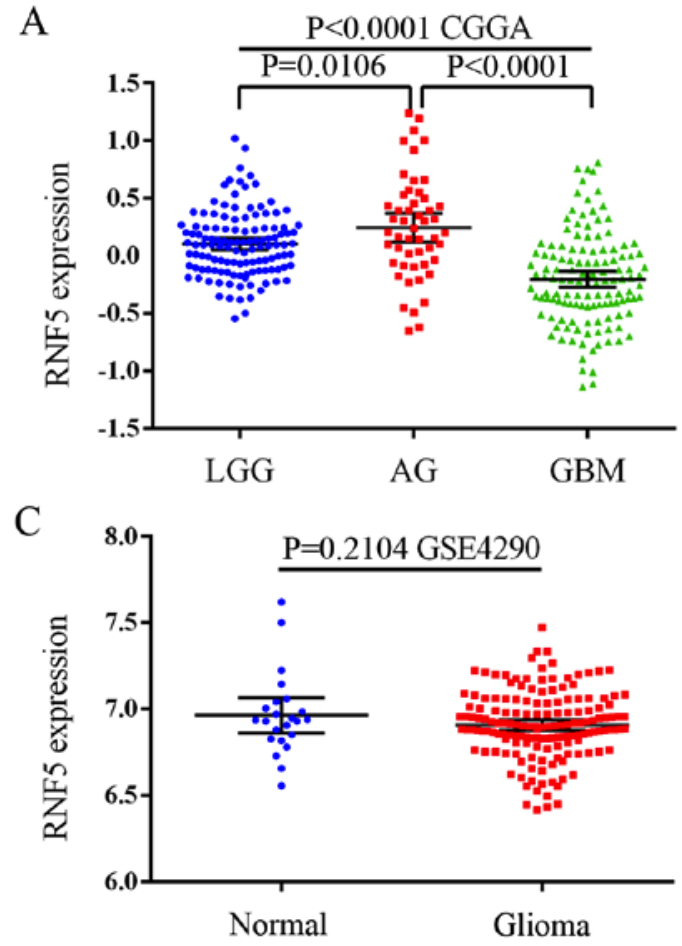

E

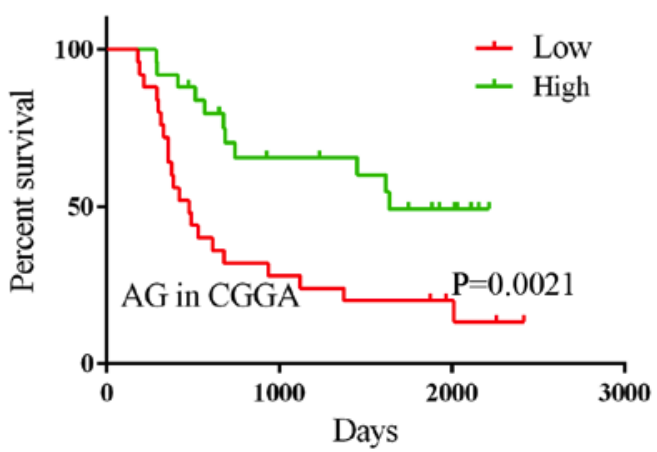

B

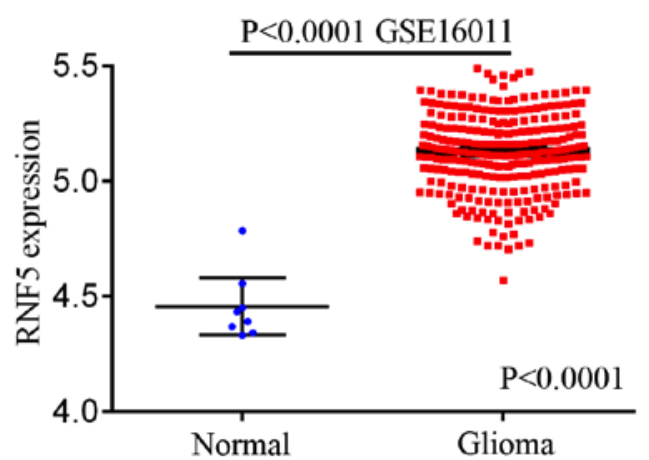

$\mathrm{D}$

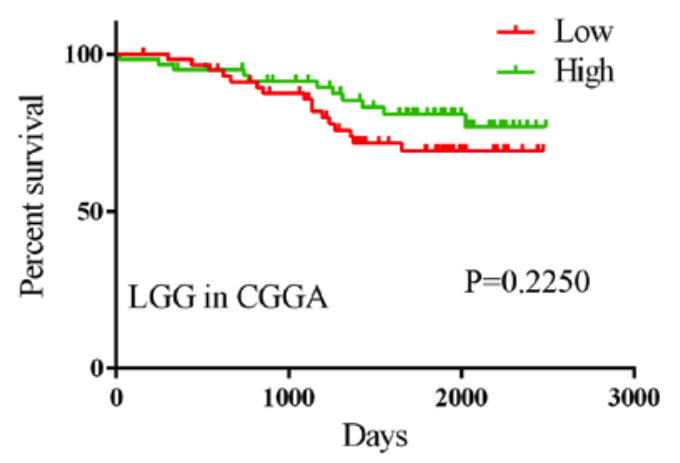

$\mathrm{F}$

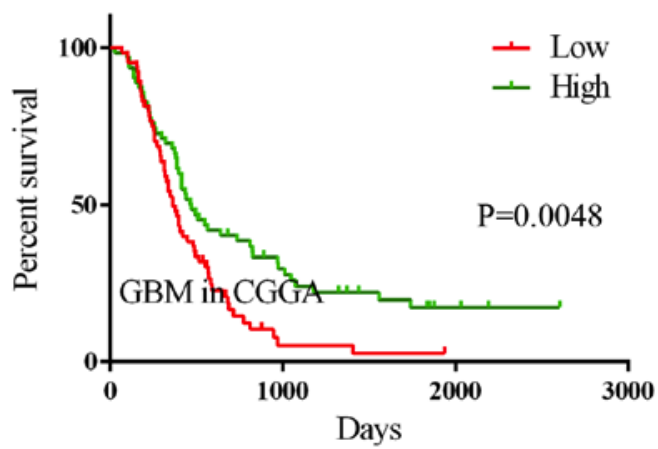

Figure 1. Expression levels of RNF5 in non-cancerous brain tissue and glioma samples from patients with different disease grades and association with patient prognosis. (A) RNF5 was differentially expressed in different grades of glioma and exhibited the highest and lowest expression in AG and GBM, respectively. (B) RNF5 expression significantly differed between non-cancerous brain and glioma tissues in the GSE16011 dataset. (C) Expression of RNF5 did not significantly differ between non-cancerous brain tissue and glioma tissues in the GSE4290 dataset. Kaplan-Meier survival curve analysis of the prognostic significance of RNF5 expression in patients with (D) LGG, (E) AG and (F) GBM. RNF5, ring finger protein 5; AG, anaplastic glioma; GBM, glioblastoma multiforme; LGG, low-grade glioma; CGGA, Chinese Glioma Genome Atlas.

(Fig. 1A). The GSE16011 and GSE4290 datasets were selected to investigate the difference in RNF5 expression between non-cancerous brain tissue and glioma; however, a consistent conclusion was not reached owing to the small number of non-cancerous brain tissue samples in the datasets (Fig. 1B and C). However, an association between RNF5 expression levels and patient prognosis was determined using the CGGA database. In LGG, the expression level of RNF5 and patient prognosis were not significantly associated, while in AG and GBM, patients with high RNF5 expression had an improved prognosis compared with patients with low expression (Fig. 1D-F).

To further investigate the association between high RNF5 expression and prognosis in patients with glioma, tumor protein 53 (TP53) mutations were analyzed as indicated by the literature (18-21). An analysis of TCGA database revealed that TP53 has a high mutation rate in GBM, while only two RNF5 mutations were identified (Fig. 2A). Additionally, in vitro analysis revealed that cells overexpressing RNF5 exhibited increased colony formation compared with control cells (Fig. 2B-D). Subsequently, the role of RNF5 in glioma and its possible target proteins were further analyzed using a bioinformatics approach.

GSEA of KEGG signaling pathways associated with RNF5 in human glioma. To further analyze the role of RNF5 in human glioma, GSEA of the CGGA database was performed. The expression levels of RNF5 in the samples were sorted from low to high, and the samples were divided into four groups: A, B, C and D. Group A contained samples with low expression of RNF5, while Group D contained samples with a high expression level of RNF5. The newly grouped data were subsequently analyzed by GSEA. The GSEA revealed that $R N F 5$ was significantly associated with 
A
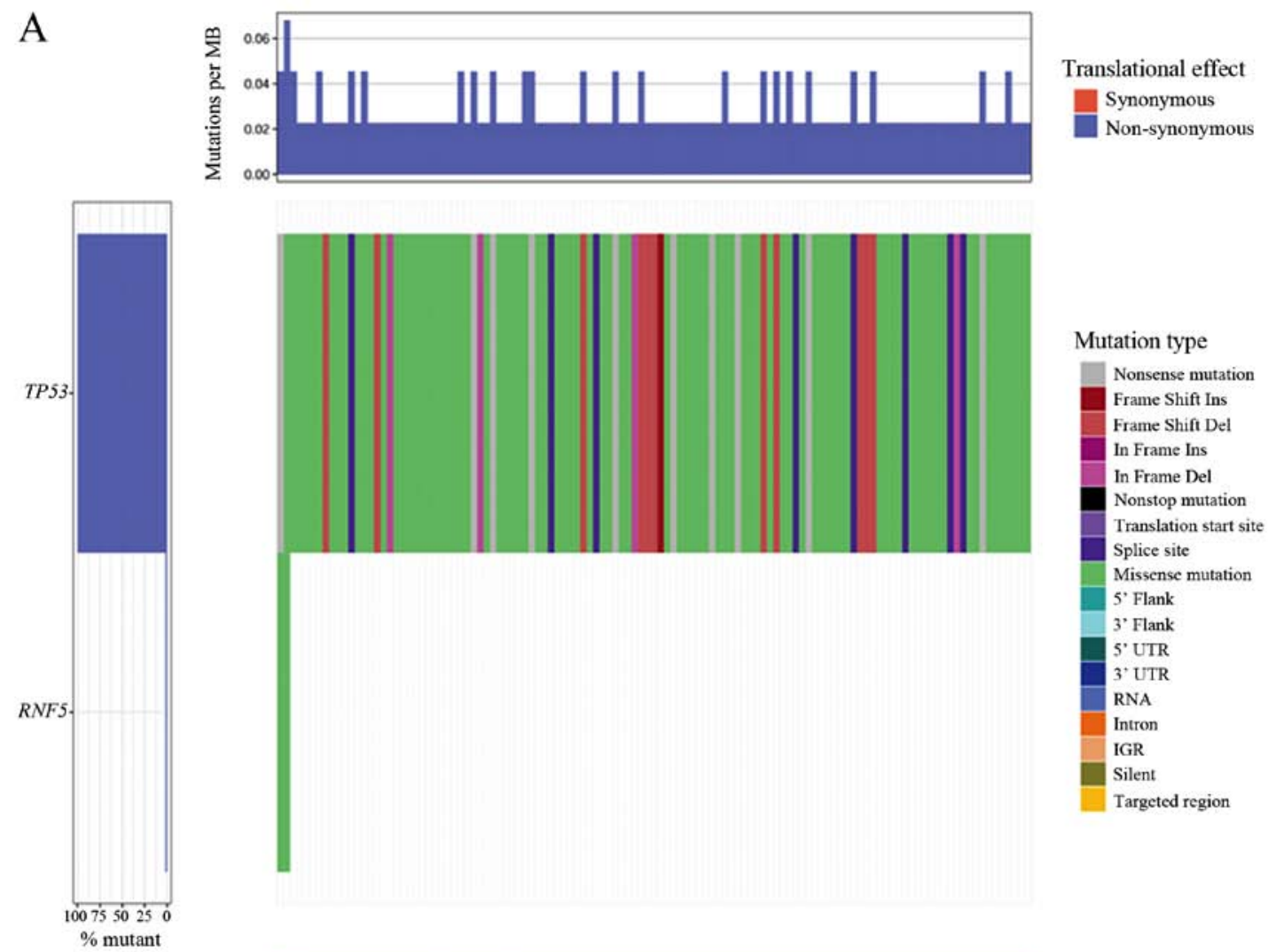

B

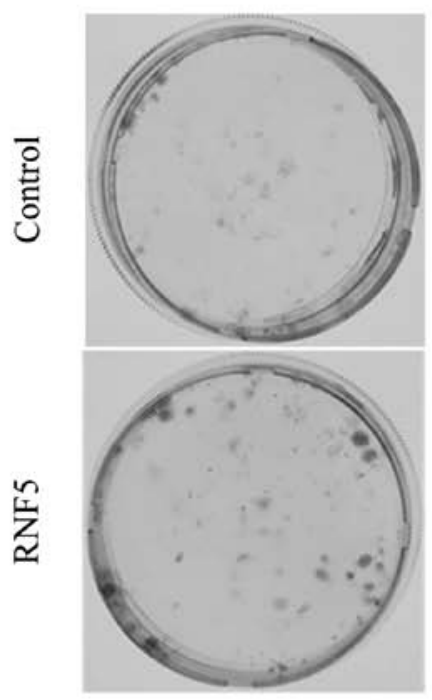

$\mathrm{C}$

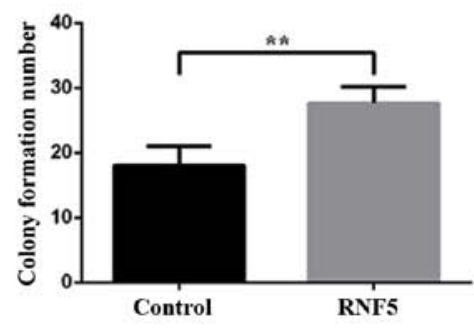

$\mathrm{D}$

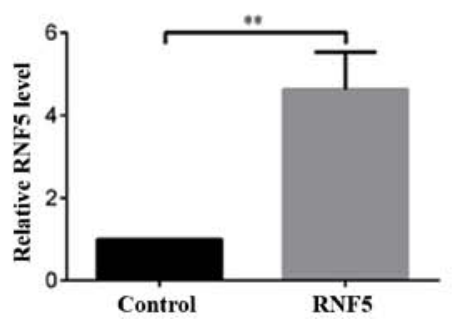

Figure 2. RNF5 and TP53 mutations identified in low-grade glioma and GBM and colony formation assay. (A) A total of 117 mutations in TP53 and two mutations in RNF5 in GBM were identified in The Cancer Genome Atlas. (B) Cell colony forming ability was increased in U251 cells overexpressing RNF5 compared with control cells. (C) Cell colony formation analysis. (D) Reverse transcription-quantitative PCR analysis of the expression levels of RNF5 in U251 cells overexpressing RNF5 compared with control cells. ${ }^{* *} \mathrm{P}<0.01$. RNF5, ring finger protein 5; TP53, tumor protein 53; 3'UTR, 3'untranslated region; GBM, glioblastoma multiforme.

the following KEGG signaling pathways: 'Wnt signaling pathway', 'apoptosis', 'cell adhesion molecules CAMs', 'cytokine-cytokine receptor interaction', 'focal adhesion' and 'ECM-receptor interaction' (Fig. 3). Therefore, RNF5 may affect the development of glioma through these KEGG signaling pathways.
Prediction of RNF5 ubiquitination substrates using a bioinformatics approach. In order to identify the potential ubiquitination substrates of RNF5 in human glioma, CGGA, GSE16011 and GSE4290 data were divided into groups of low and high RNF5 expression. Differential genetic analysis was then performed on these groups to identify the DEGs. 
A

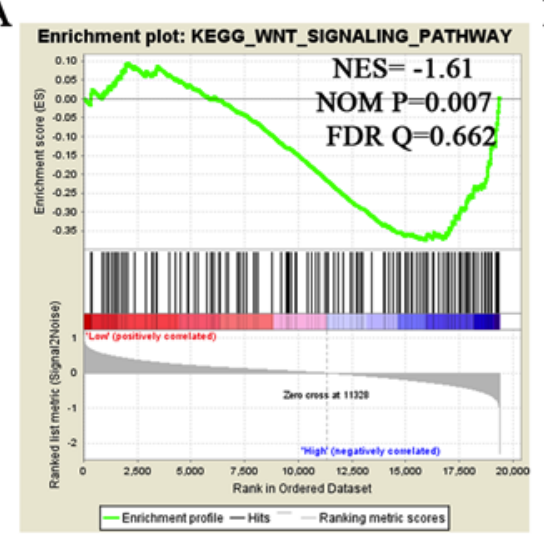

D

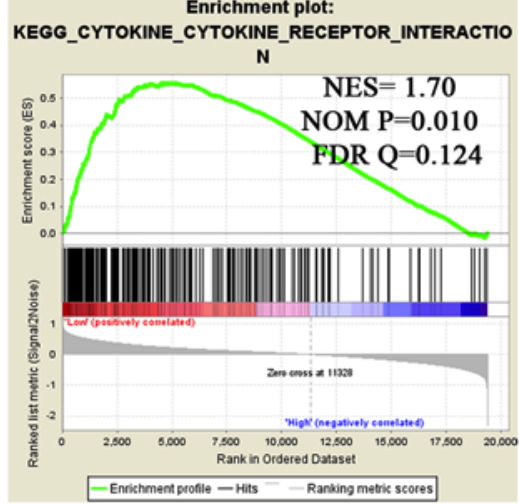

B

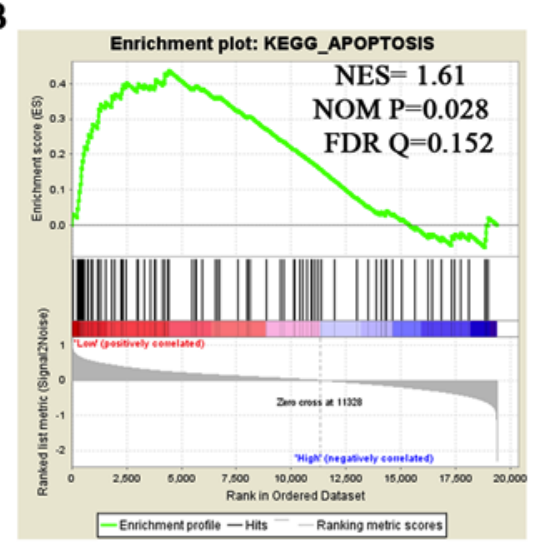

E

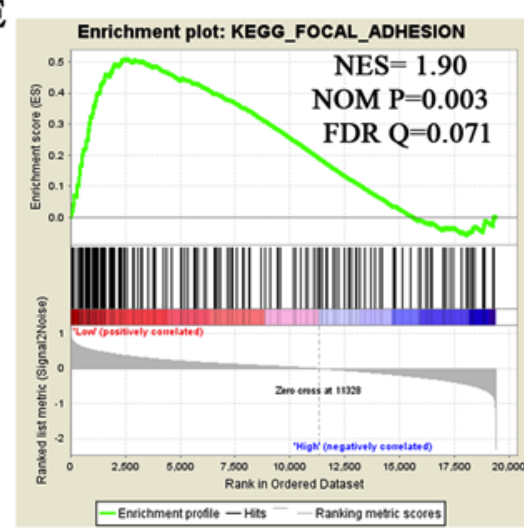

C

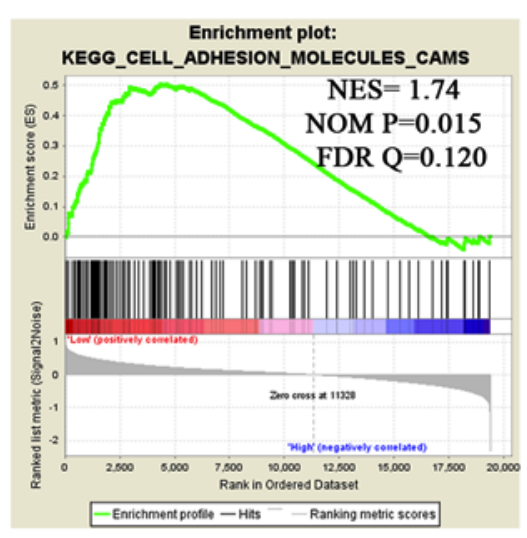

F

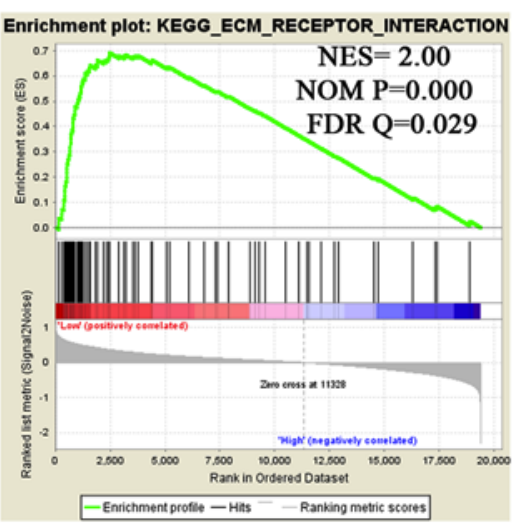

Figure 3. GSEA to identify significant $R N F 5$-enriched KEGG signaling pathways. GSEA revealed that RNF5 is enriched in the following pathways: (A) The 'Wnt signaling pathway', (B) 'apoptosis', (C) 'cell adhesion molecules CAMs', (D) 'cytokine-cytokine receptor interaction', (E) 'focal adhesion' and (F) 'ECM-receptor interaction'. GSEA, Gene Set Enrichment Analysis; RNF5, ring finger protein 5; KEGG, Kyoto Encyclopedia of Genes and Genomes; ECM, extracellular matrix; NES, normalized enrichment score; NOM, nominal; FDR, false discovery rate.

A

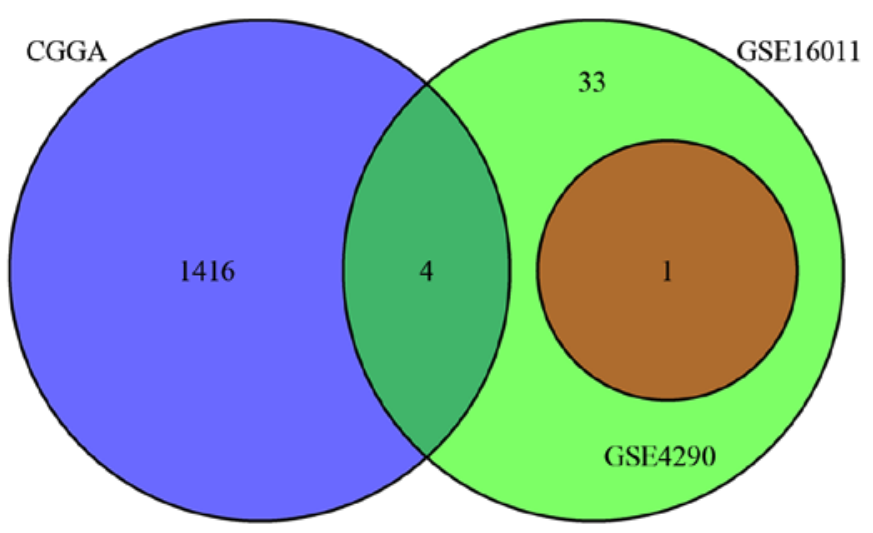

B

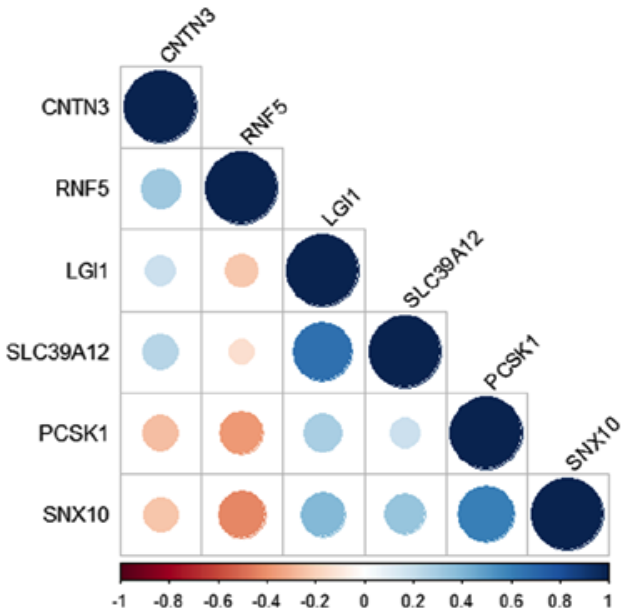

Figure 4. RNF5 ubiquitin substrate prediction. (A) Venn diagram showing 4 overlapping genes between the CGGA database and the GSE16011 dataset and 1 overlapping gene between the GSE4290 and GSE16011 datasets. (B) Associations between RNF5 and these 5 genes. Red and blue dots indicate a negative and positive association, respectively. The greater the size and color intensity of the dot, the stronger the association. $R N F 5$, ring finger protein 5 ; CGGA, Chinese Glioma Genome Atlas; CNTN3, contactin 3; LGI1, leucine rich glioma inactivated 1; SLC39A12, solute carrier family 39 member 12; PCSK1, proprotein convertase subtilisin/kexin type 1 ; SNX10, sorting nexin 10 .

To further clarify the possible ubiquitination substrates of $R N F 5$, the overlapping genes between the CGGA database and GSE16011 and GSE4290 datasets were identified (Fig. 4A). A total of 4 overlapping genes were identified between the CGGA database and the GSE16011 dataset, 1 overlapping gene was identified between the GSE16011 and
GSE4290 datasets, and no overlapping genes were identified between the CGGA database and the GSE4290 dataset. The 5 overlapping genes included contactin 3 (CNTN3), leucine rich glioma inactivated $1(L G I 1)$, proprotein convertase subtilisin/kexin type 1 ( $P C S K 1)$, sorting nexin 10 (SNX10) and solute carrier family 39 member 12 (SLC39A12; Fig. 4B). 
A

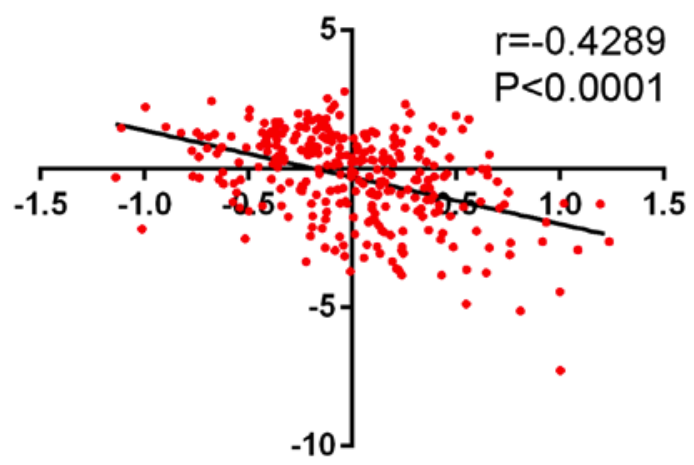

C LGI1 correlations

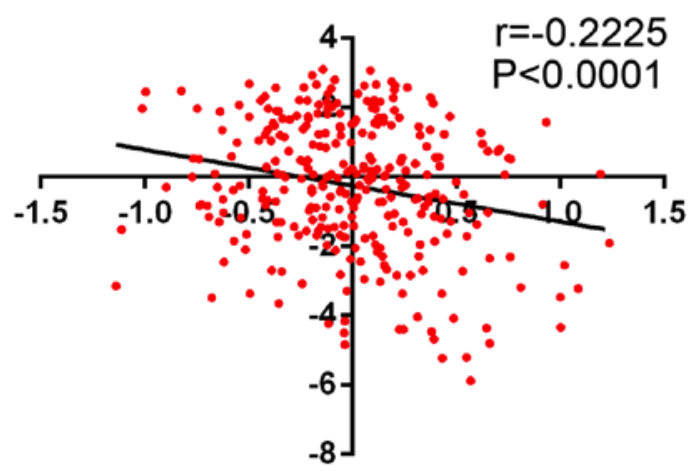

B PCSK1 correlations

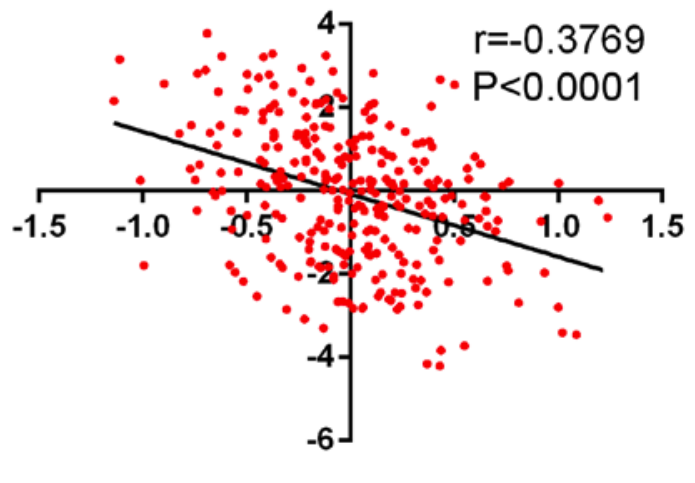

D SLC39A12 correlations

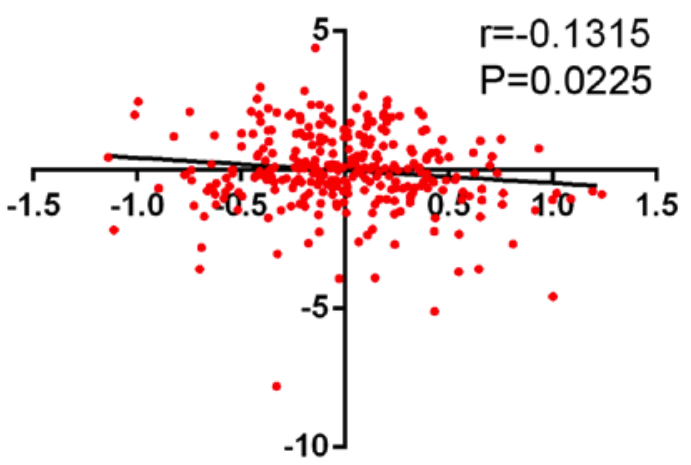

Figure 5. Correlation between RNF5 and the 4 negatively correlated genes. Correlation between RNF5 and (A) SNX10, (B) PCSK1, (C) LGI1 and (D) SLC39A12. $R N F 5$, ring finger protein $5 ; S N X 10$, sorting nexin 10; PCSK1, proprotein convertase subtilisin/kexin type 1; LGI1, leucine rich glioma inactivated 1; SLC39A12, solute carrier family 39 member 12 .

RNF5 expression was positively associated with CNTN3, while a negative association was demonstrated for the remaining four genes (SNX10, PCSK1, LGI1 and SLC39A12) (Fig. 4B). In addition, the results from Fig. 5 demonstrated that RNF5 was negatively correlated with SNX10, PCSK1, LGI1 and SLC39A12.

\section{Discussion}

Previous studies have demonstrated that ubiquitin ligase is closely associated with tumor development and metastasis $(13,22-25)$. The present study used human glioma data to reveal that RNF5 was differentially expressed in patients with different levels of glioma and was correlated with prognosis in patients with AG and GBM. Specifically, an improved prognosis was observed in patients with AG and GBM with a high expression of RNF5 compared with a low expression. Subsequently, RNF5 was overexpressed in U251 cells in vitro, and it was revealed that colony forming ability was enhanced in cells overexpressing RNF5 compared with controls. The authors speculate that silencing $R N F 5$ reduces the colony forming ability. Through GSEA enrichment analysis, KEGG signaling pathways that were significantly associated with $R N F 5$ were identified. To further explore possible ubiquitination substrates for $R N F 5$ in human glioma, correlation analysis were performed. A total of 4 genes were negatively associated with $R N F 5$ expression, and may serve as potential RNF5 ubiquitination substrates.

Previous studies revealed that RNF5 was highly expressed in breast cancer specimens and cell lines. Additionally, tumor cell proliferation was inhibited after silencing $R N F 5$, and patients with breast cancer with high RNF5 expression have a poor prognosis compared with patients with low expression $(13,21)$. Cell proliferation was inhibited after silencing RNF5 expression in MCF-7 cells. However, cell proliferation was not affected in MDA-MB-231, MDA-MB-435 and BT-474 cells following RNF5 silencing due to differences in TP53 status, as TP53 is only functional in MCF-7 cells $(21,26,27)$. Furthermore, TP53 expression was increased following the silencing of RNF5 in MCF-7 cells, suggesting that RNF5 may be involved in the inhibition of TP53 by Rho GTPase, Src networks or ERAD (21). The present study demonstrated that higher RNF5 expression was associated with an improved prognosis in patients with glioma, which may be consistent with TP53 mutations in glioma, particularly in GBM $(28,29)$. Previous studies revealed a higher rate of TP53 mutations in patients with glioma compared with healthy subjects (30-33). Therefore, it is possible that in patients with glioma, mutations in TP53 may reverse the inhibition of proliferation induced by $R N F 5$ silencing. The apparently opposite prognostic effect of $R N F 5$ expression levels in patients with glioma necessitates further investigation of the mechanism of action of RNF5. The 
present study investigated cell colony formation of U251 cells overexpressing RNF5. However, experiments on apoptosis, invasion and migration should be performed in future studies. Furthermore, the lack of validation on clinical samples is a limitation of the present study.

In summary, the present study revealed that RNF5 is differentially expressed in patients with glioma with different disease grades and is associated with patient prognosis. Moreover, GSEA revealed KEGG pathways that are significantly associated with RNF5. Through correlation analysis, possible ubiquitin substrates for RNF5 in patients with glioma were predicted. These results provided a meaningful insight into the treatment of glioma.

\section{Acknowledgements}

The authors would like to thank Mr. Xue Shengbai (Clinical Department of Nanjing Medical University) for providing technical support.

\section{Funding}

The present study was supported by the Foundation of Jiangsu Provincial Health Department (grant no. YG201514), Xuzhou Municipal Bureau on Science and Technology (grant no. XM12B055) and Xuzhou Medical University (grant no. 2018KJ09).

\section{Availability of data and materials}

The datasets used and/or analyzed during the current study are available from the corresponding author on reasonable request.

\section{Authors' contributions}

YG, CX, MJ, QS and YS conceived and designed this study. YG, CX, MJ, QA, BZ, XC, LW and YW performed the experiments. YG, CX and LW conducted statistical analysis. YG, CX, QA, QS and YS wrote the manuscript. All authors read and approved the final manuscript.

\section{Ethics approval and consent to participate}

The present study protocol was approved by the Ethics Committee of Xuzhou Children's Hospital.

\section{Patient consent for publication}

Not applicable.

\section{Competing interests}

The authors declare that they have no competing interests.

\section{References}

1. Stupp R and Roila F; ESMO Guidelines Working Group: Malignant glioma: ESMO clinical recommendations for diagnosis, treatment and follow-up. Ann Oncol 20 (Suppl 4): S126-S128, 2009.

2. Meyer MA: Malignant gliomas in adults. N Engl J Med 359: 1850,2008
3. Stupp R, Hegi ME, van den Bent MJ, Mason WP, Weller M, Mirimanoff RO and Cairncross JG; European Organisation for Research and Treatment of Cancer Brain Tumor and Radiotherapy Groups; National Cancer Institute of Canada Clinical Trials Group: Changing paradigms-an update on the multidisciplinary management of malignant glioma. Oncologist 11: 165-180, 2006.

4. Stupp R, Hottinger AF, van den Bent MJ, Dietrich PY and Brandes AA: Frequently asked questions in the medical management of high-grade glioma: A short guide with practical answers. Ann Oncol 19 (Suppl 7): vii209-vii216, 2008.

5. Buonerba C, Di Lorenzo G, Marinelli A, Federico P, Palmieri G, Imbimbo M, Conti P, Peluso G, De Placido S and Sampson JH: A comprehensive outlook on intracerebral therapy of malignant gliomas. Crit Rev Oncol Hematol 80: 54-68, 2011.

6. Sherman JH, Hoes K, Marcus J, Komotar RJ, Brennan CW and Gutin PH: Neurosurgery for brain tumors: Update on recent technical advances. Curr Neurol Neurosci Rep 11: 313-319, 2011.

7. Kyushiki H, Kuga Y, Suzuki M, Takahashi E and Horie M: Cloning, expression and mapping of a novel RING-finger gene (RNF5), a human homologue of a putative zinc-finger gene from Caenorhabditis elegans. Cytogenet Cell Genet 79: 114-117, 1997.

8. Matsuda N, Suzuki T, Tanaka K and Nakano A: Rmal, a novel type of RING finger protein conserved from Arabidopsis to human, is a membrane-bound ubiquitin ligase. J Cell Sci 114: 1949-1957, 2001.

9. Younger JM, Chen L, Ren HY, Rosser MF, Turnbull EL, Fan CY, Patterson C and Cyr DM: Sequential quality-control checkpoints triage misfolded cystic fibrosis transmembrane conductance regulator. Cell 126: 571-582, 2006.

10. Grove DE, Fan CY, Ren HY and Cyr DM: The endoplasmic reticulum-associated Hsp40 DNAJB12 and Hsc70 cooperate to facilitate RMA1 E3-dependent degradation of nascent CFTRDeltaF508. Mol Biol Cell 22: 301-314, 2011.

11. Didier C, Broday L, Bhoumik A, Israeli S, Takahashi S, Nakayama K, Thomas SM, Turner CE, Henderson S, Sabe H and Ronai Z: RNF5, a RING finger protein that regulates cell motility by targeting paxillin ubiquitination and altered localization. Mol Cell Biol 23: 5331-5345, 2003.

12. Zhong B, Zhang L, Lei C, Li Y, Mao AP, Yang Y, Wang YY, Zhang XL and Shu HB: The ubiquitin ligase RNF5 regulates antiviral responses by mediating degradation of the adaptor protein MITA. Immunity 30: 397-407, 2009.

13. Jeon YJ, Khelifa S, Ratnikov B, Scott DA, Feng Y, Parisi F, Ruller C, Lau E, Kim H, Brill LM, et al: Regulation of glutamine carrier proteins by RNF5 determines breast cancer response to ER stress-inducing chemotherapies. Cancer Cell 27: 354-369, 2015.

14. Gao Y, Han D, Sun L, Huang Q, Gai G, Wu Z, Meng W and Chen X: PPAR $\alpha$ regulates the proliferation of human glioma cells through miR-214 and E2F2. Biomed Res Int 2018: 3842753 , 2018.

15. Gravendeel LA, Kouwenhoven MC, Gevaert O, de Rooi JJ, Stubbs AP, Duijm JE, Daemen A, Bleeker FE, Bralten LB, Kloosterhof NK, et al: Intrinsic gene expression profiles of gliomas are a better predictor of survival than histology. Cancer Res 69: 9065-9072, 2009.

16. Sun L, Hui A, Su Q, Vortmeyer A, Kotliarov Y, Pastorino S, Passaniti A, Menon J, Walling J, Bailey R, et al: Neuronal and glioma-derived stem cell factor induces angiogenesis within the brain. Cancer Cell 9: 287-300, 2006.

17. Livak KJ and Schmittgen TD: Analysis of relative gene expression data using real-time quantitative PCR and the 2(-Delta DeltaC(T)) method. Methods 25: 402-408, 2001.

18. Zhang X, Tian Q, Wang L, Liu Y, Li B, Liang Z, Gao P, Zheng K, Zhao B and Lu H: Radiomics strategy for molecular subtype stratification of lower-grade glioma: Detecting IDH and TP53 mutations based on multimodal MRI. J Magn Reson Imaging 48: 916-926, 2018.

19. Lehrer S, Rheinstein PH, Green S and Rosenzweig KE: von Willebrand factor gene expression in primary lower grade glioma: Mutually Co-occurring mutations in von Willebrand factor, ATRX, and TP53. Brain Tumor Res Treat 7: 33-38, 2019.

20. Malmer B, Gronberg H, Andersson U, Jonsson BA and Henriksson R: Microsatellite instability, PTEN and p53 germline mutations in glioma families. Acta Oncol 40: 633-637, 2001.

21. Bromberg KD, Kluger HM, Delaunay A, Abbas S, DiVito KA, Krajewski S and Ronai Z: Increased expression of the E3 ubiquitin ligase RNF5 is associated with decreased survival in breast cancer. Cancer Res 67: 8172-8179, 2007. 
22. Shi H, Zheng B, Wu Y, Tang Y, Wang L, Gao Y, Gong H, Du J and Yu R: Ubiquitin ligase Siah1 promotes the migration and invasion of human glioma cells by regulating HIF-1 $\alpha$ signaling under hypoxia. Oncol Rep 33: 1185-1190, 2015.

23. Wu Y, Wang L, Bao H, Zou S, Fu C, Gong H, Gao Y, Tang Y, Yu R and Shi H: Nrdp1S, short variant of Nrdp1, inhibits human glioma progression by increasing Nrdp1-mediated ErbB3 ubiquitination and degradation. J Cell Mol Med 20: 422-429, 2016

24. Shi H, Du J, Wang L, Zheng B, Gong H, Wu Y, Tang Y, Gao Y and Yu R: Lower expression of Nrdp1 in human glioma contributes tumor progression by reducing apoptosis. IUBMB Life 66 704-710, 2014.

25. Shi H, Gong H, Cao K, Zou S, Zhu B, Bao H, Wu Y, Gao Y, Tang Y and Yu R: Nrdp1-mediated ErbB3 degradation inhibits glioma cell migration and invasion by reducing cytoplasmic localization of p27(Kip1). J Neurooncol 124: 357-364, 2015.

26. Runnebaum IB, Nagarajan M, Bowman M, Soto D and Sukumar S: Mutations in p53 as potential molecular markers for human breast cancer. Proc Natl Acad Sci USA 88: 10657-10661, 1991.

27. Nieves-Neira W and Pommier Y: Apoptotic response to camptothecin and 7-hydroxystaurosporine (UCN-01) in the 8 human breast cancer cell lines of the NCI Anticancer Drug Screen: Multifactorial relationships with topoisomerase I, protein kinase $\mathrm{C}, \mathrm{Bcl}-2$, p53, MDM-2 and caspase pathways. Int J Cancer 82: 396-404, 1999.
28. Kawasoe T, Takeshima H, Yamashita S, Mizuguchi S, Fukushima T, Yokogami K and Yamasaki K: Detection of p53 mutations in proliferating vascular cells in glioblastoma multiforme. J Neurosurg 122: 317-323, 2015.

29. Marutani M, Tonoki H, Tada M, Takahashi M, Kashiwazaki H, Hida Y, Hamada J, Asaka M and Moriuchi T: Dominant-negative mutations of the tumor suppressor p53 relating to early onset of glioblastoma multiforme. Cancer Res 59: 4765-4769, 1999.

30. Lin C, Liang Y, Zhu H, Zhang J and Zhong X: R280T mutation of p53 gene promotes proliferation of human glioma cells through GSK-3ß/PTEN pathway. Neurosci Lett 529: 60-65, 2012.

31. Robertson LB, Armstrong GN, Olver BD, Lloyd AL, Shete S, Lau C, Claus EB, Barnholtz-Sloan J, Lai R, Il'yasova D, et al: Survey of familial glioma and role of germline p16INK4A/p14ARF and p53 mutation. Fam Cancer 9: 413-421, 2010

32. Cui W, Wu R, Cao H, Gao J, Wang X and Ren Q: P53 gene mutation and expression of MDM2, P53, P16 protein and their relationship in human glioma. J Huazhong Univ Sci Technolog Med Sci 25: 622-624, 635, 2005.

33. Hayashi Y, Yamashita J and Yamaguchi K: Timing and role of p53 gene mutation in the recurrence of glioma. Biochem Biophys Res Commun 180: 1145-1150, 1991.

This work is licensed under a Creative Commons Attribution-NonCommercial-NoDerivatives 4.0 International (CC BY-NC-ND 4.0) License. 